\title{
Reale und fiktive Sender-Adressaten-Konstellationen in Fußball-Fangesängen - mit romanistischen Beispielen
}

\author{
Eva LAVRIC (Innsbruck) ${ }^{1}$
}

\section{Summary}

Football chants have been researched in several disciplines - from musicology to ethnology to linguistics for quite a while now. They have been most intensively investigated in the German speaking countries, thus focusing on German chants; Kopiez/Brink 1998 is still the reference work in this respect. Other languages and cultures, however, have been studied much less; this paper therefore proposes an investigation of the (lyrics of) football chants of Romance language cultures using a corpus of French, Spanish and Italian songs. The analysis follows a new categorizing scheme, namely "participant constellations", i.e. constellations of senders and addressees (fans towards their own team, fans towards referees, fans towards opponents' fans etc.). The results reveal that constellations can be both real and fictitious; in the latter case, the fans sing a text which takes the position of a first person and is sung from the perspective of one exemplary fan. This is the case in chants which stylize the fan's connection with his/her team as a romantic relationship. The corpus also contains other, sometimes very original fictitious speakers that are linked to the feeling of togetherness among the fan base and show creativity and humour.

Fußball-Fangesänge sind in der Forschung - sei sie nun musikwissenschaftlich, ethnologisch, kulturwissenschaftlich oder linguistisch ${ }^{2}$ - schon länger keine Unbekannten mehr. Besonders intensiv war die Beschäftigung damit im deutschsprachigen Raum und daher mit deutschen Chants $^{3}$, wobei als Standardwerk immer noch Kopiez/Brink 1998 zu nennen ist. In diesem Rahmen entstanden auch Kategorisierungsversuche wie der von Khodadadi/ Gründel 2006 nach Funktionen/Intentionen (von unterstützend über euphorisch bis diffamierend) und der sehr empfehlenswerte von Burkhardt 2009 nach Sprechakttypen (Direktiva, Assertiva und Expressiva, jeweils mit Untertypen). Unterbeleuchtet blieben dabei allerdings andere Sprachen und Kulturen, insbesondere die romanischen (es gibt nur Minonzio 2014 für Französisch und Mühlbacher 2011 für Spanisch ${ }^{4}$ ), so dass sich der vorliegende Beitrag zur Aufgabe stellt, die (Texte der) Fangesänge der romanischen Kulturräume anhand eines Korpus aus französischen, spanischen und italienischen Chants zu untersuchen. Gleichzeitig soll dabei eine neue Kategorisierung erprobt werden, und zwar nach participation frame- 
works bzw. participant constellations, also nach Sender-Adressaten-Konstellationen (Fans an eigene Mannschaft, Fans an Schiedsrichter, Fans an gegnerische Fans etc.). Dabei wird sich zeigen, dass neben den realen Konstellationen auch fiktive auftreten, bei denen die Gruppe der Fans einen Text singt, der in Ich-Form einem einzelnen, exemplarischen Fan in den Mund gelegt wird; dies geschieht vor allem in solchen Gesängen, in denen der Bezug des Fans zu seiner Mannschaft als Liebesbeziehung stilisiert wird. Daneben werden aber auch noch andere, fiktive Sprecher*innen bzw. auch Adressat*innen in Szene gesetzt, wobei sich die Kreativität und der Humor der Fangemeinde entfalten können. Insgesamt lassen sich aus den Teilnehmer-Konstellationen Rückschlüsse auf die Art der Identitätskonstruktion und der Herstellung von Zusammengehörigkeit in der Fangemeinde ziehen.

Präludierend wollen wir uns aber mit den moralischen Fragen auseinandersetzen, die Fangesänge durchaus auch aufwerfen können.

\section{Sind Fußball-Fangesänge gut oder böse?}

Fußball-Fangesänge machen Stimmung im Stadion. Sie verwandeln Tausende Einzelne in einen großen Klangkörper. Sie brechen die Grenze zwischen Akteuren und Zuschauern auf: Das Publikum wird zum ,zwölften Mann', es hat aktiv teil am Geschehen, das es wie der antike $\mathrm{Chor}^{5}$ begleitet und kommentiert. Fußball-Fangesänge sind aber nicht nur freundlich, sondern auch gemein. Denn sie sind parteiisch. Sie trennen die Welt in ein ,Wir ' und ,die anderen'. ${ }^{6}$ Sie drohen dem Gegner, sie beleidigen ihn. Fußball-Fangesänge sind so, wie die Welt ist: gut und böse zugleich.

,Gut' sind Fangesänge, weil sie ein Zusammengehörigkeitsgefühl ausdrücken, das für viele Menschen Heimat ist; weil sie Engagement, Kreativität und Humor verströmen; und weil sie kollektive Emotionen im gemeinsamen Singen (und Agieren) intensiv erlebbar machen, in einem Setting, das stark ritualisiert ist und für die Insider spirituell-religiösen Charakter annimmt. Dieser Gedanke des Fußballs als Religion kommt in der gesamten Fachliteratur immer wieder vor (cf. z. B. Rühlemann 1996, Kopiez 2002, Schmidt-Lauber 2009); ausführlich begründet und illustriert wird er von Bromberger 1995 und 1998. Es geht dabei vor allem um den Ritualcharakter und um die Sakralisierung des Fußballsports, zu der das Singen im Chor einen wichtigen Beitrag leistet.

Trotzdem sind Fangesänge auch ,böse', weil diese Zusammengehörigkeit, die sie stiften, in ihrem tiefsten Wesen auf einer Gegnerschaft aufbaut - einer momentanen Gegnerschaft, am Tag des Spiels; aber oft auch auf langwährenden Rivalitäten zwischen bestimmten Klubs einer Stadt oder eines Landes, wie sie sich im Derby äußern. ${ }^{7}$ In diesem Sinne beschreibt Luhrs 2009 Fangesänge als Erben der uralten blason populaire-Tradition: Dabei handelt es sich um Sprüche, Reime und Lieder, bzw. auch Spottnamen und Scherze, mit denen sich die Bewohner benachbarter Dörfer/Orte/Städte gegenseitig schlechtmachen bzw. beleidigen und so gleichzeitig ihre jeweilige Wir-Identität stärken. Blason populaire ist also "[a]n expression of one’s group 
outlook and self-image, often involving the implied simultaneous detraction and/or detriment of another (rival) group“" (Green/Widdowson 2003, 9, zitiert nach Luhrs 2009, 234).

Vanhoutte 2018 bringt das Wesen des Fußballderbys mit der Relevantsetzung minimaler Unterschiede in Nachbarschaftsrivalitäten in Zusammenhang, die er nach dem Vorbild von Freuds „Narzissmus der kleinen Differenzen“ interpretiert; er spricht von einer subtilen Verbindung von Liebe und Hass, die ausschließlich auf winzigen Differenzen basiert, die von den Fans in narzisstischer Weise betont und auf diese Weise emotional aufgeladen werden (cf. Vanhoutte 2018, 128, 134 und 137).

Trotzdem: Auch in solchen willkürlichen Feindschaften entsteht Heimat, entsteht Gruppenidentität. Damit stehen die Erzrivalen in einem sehr engen Bezug zueinander, ein jeder definiert sich in hohem Maß auch als Gegner, als Konkurrent des anderen. Daher ist es auch in gewisser Weise so, dass sie einander brauchen. Sehr schön herausgearbeitet hat das Vejmelka (2016), der die Rivalität zwischen Real Madrid und Barcelona anhand zweier berühmter Autoren illustriert, Javier Marías als merengue für Barça und Manuel Vázquez Montalbán als culé für Real Madrid: Irgendwann bekennen beide, dass ihnen der Erzrivale über die Jahre ans Herz gewachsen ist und dass der Fußball und die eigene Fanidentität ohne die jeweils andere Mannschaft gar nicht mehr denkbar wären. Ein dialektischer Zusammenhang also, den man leben und doch gleichzeitig durchschauen kann.

$\mathrm{Ob}$ das die böse gemeinten, feindlichen Fangesänge verzeihlich oder zumindest verständlich macht? Für manche wird das wohl so sein, auch angesichts der Tatsache, dass sich die Fangemeinden gegenseitig nichts schenken - Meuren 2006 interpretiert das als eine rituelle Zerstörung des Gegners, die das ,Ich' und ,Wir' der Fangemeinde und der eigenen Mannschaft erhöhen soll. Für andere erscheint es trotzdem weiterhin seltsam, wie sonst friedlich und zuvorkommend sozialisierte Menschen im Stadion, im Schutz der Masse und von ihr mitgerissen, plötzlich eine bösartige, hasserfüllte Seite hervorkehren. Das zeigt sich auch darin, dass in der Football-Chant-Forschung immer wieder einzelne Artikel gerade diese Lieder hervorheben und kommentieren, cf. Bastian/Koch 2015 und insbesondere Küster:

Die Metaphorik der Kampflieder und Schlachtgesänge ist häufig von archaischer Einfachheit. Tier und Mensch rücken verbal aufs Engste zusammen. Besonders deftig ist die Metaphorik dort, wo in sogenannten Derbys Fangruppen in Fußballstadien aufeinandertreffen, die im sonstigen Leben in derselben Region, also gewissermaßen in nachbarschaftlicher Zwietracht, irgendwo zwischen Dortmund und Gelsenkirchen Tür an Tür wohnen, oder die sich sogar eine Stadt teilen müssen, sei es nun Hamburg, Leipzig, München oder Berlin. (Küster 2009, 75)

Seltsam mögen auch die Inhalte der positiven („solidarischen“ oder „euphorischen“, cf. Khodadadi/Gründel 2006, 10-11 bzw. unten im Analysekapitel) Fangesänge anmuten, in denen die Fans ,ihrem 'Verein ihre Unterstützung, Liebe und ewige Treue versichern. Die Ebene der Fans unterscheidet sich dabei nämlich ganz grundlegend von jener der Spieler: Während das Wesentliche an einem Fan die Treue, die ewige Loyalität zu seinem Verein 
ist (,durch dick und dünn'), die er in etlichen Gesängen immer wieder bekräftigt, wechseln die Spieler und auch die Trainer locker von einem Klub zum anderen, ohne dass ihnen das (im Allgemeinen) übelgenommen wird, denn das gehört eben zu ihrem Beruf. Dadurch kann es vorkommen, dass sie gegen den früheren eigenen Verein spielen und dabei beweisen müssen, dass sie ihre Loyalität und Treue innerhalb kurzer Zeit völlig umgepolt haben. Für diese Art von ,Treuebruch ' haben die Fans durchaus Verständnis, jedenfalls in ihren Chants wird er in der Regel nicht geahndet. ${ }^{8}$

Ein zweiter wichtiger Unterschied zwischen Fans und Spielern besteht darin, dass die Spieler den Fußball als Sport betreiben, die Fans aber, wie bereits angemerkt, als Religion. Im Sport wird Fair Play und Rücksicht auf den Gegner großgeschrieben. Die Spieler der gegnerischen Mannschaften helfen sich nach Fouls und Stürzen gegenseitig beim Aufstehen, sie sehen von Beleidigungen weitgehend ab, und am Ende tauschen sie ihre Trikots. Damit sind sie ein Vorbild für die Jugend und verdienen die volle Sympathie. Aber die Fans? Sie fechten untereinander regelrechte Religionskriege aus, mit den Fangesängen als Kampfliedern (und mit der durchaus realen Gefahr des Ausartens in physische Gewalt). Wenn ein zigtausendstimmiger Chor die gegnerische Mannschaft bedroht oder beleidigt, kann das wohl kaum als Vorbild für den gesellschaftlichen Umgang mit konkurrierenden Gruppen angesehen werden. Es gibt nichts daran zu rütteln: Fangesänge sind ,gut' und ,böse' zugleich.

Nach diesem moralischen Prolog kommen wir zum eigentlichen Thema und werden zunächst ganz kurz auf die Fachliteratur eingehen.

\section{Literaturüberblick und Klassifizierungsversuche der Fangesänge}

Die Fachliteratur zu Fangesängen ist relativ reichhaltig, und sie beleuchtet das Phänomen aus den verschiedensten Perspektiven, von der Musikwissenschaft über die Kulturwissenschaft, Ethnologie und Soziologie bis hin zur Linguistik. Das von allen zitierte Standardwerk ist Kopiez/Brink 1998, das mit deutschen Beispielen arbeitet und eigentlich von der Musikwissenschaft herkommt. Auf dieses Werk sei auch verwiesen, wer sich für die musikalische Seite der Fangesänge interessiert, die mit ihren Entlehnungen und Vereinfachungen ein sehr spannendes Forschungsthema darstellt. In Kopiez/Brink findet man, neben einem ausgezeichneten Literaturüberblick, in handliche Kapitel eingeteilt z. B. Überlegungen über das Phänomen des Massengesangs, über den Ursprung der Fangesänge, über die bricolageTechnik mit vorgefundenen Musikstücken und neu unterlegten Texten, über die Rolle der Chants beim Match, aber auch vor und nach dem Spiel, über die Organisation bzw. Steuerung des Singens innerhalb der Fan-Gemeinschaften (mit einem chant leader, der das Repertoire pflegt und erweitert), außerdem über das Repertoire verschiedener Klubs in der deutschen Bundesliga, über die Veränderungen dieses Repertoires in der Zeit und schließlich auch darüber, was (musikalisch) einen guten Fangesang ausmacht. Das Spektrum ist so reichhaltig und umfassend, dass sich die darauffolgende Literatur sehr stark an diesem echten Standardwerk orientiert. Wir verzichten daher weitgehend auf die musikwissenschaftli- 
chen sowie kulturwissenschaftlich-ethnologischen Aspekte und heben aus den zahlreichen Titeln der Fachliteratur vor allem jene hervor, die von linguistischem Interesse sind - und unter diesen noch einmal jene, die Einteilungsversuche liefern, da wir auch selbst eine solche Klassifikation versuchen werden.

Hoffmann 2015 geht an Fußball-Chants mit einem strikt linguistisch-pragmatischen Ansatz heran, und zwar im Rahmen der Usage-based Construction Grammar. In der Tat prädestiniert die Simplizität und die Variabilität innerhalb vorgegebener Schemata FußballFangesänge für eine Deutung in diesem Rahmen. Hoffmann gibt drei englische Beispiele mit schematischer Construction-Grammar-Interpretation; eines davon soll hier wiedergegeben werden (Hoffmann 2015, 278). Es handelt sich um den Chant „Are you England? Are you England? Are you England in disguise? Are you England in disguise?", bei dem England durch jeglichen Namen eines Rivalen des eigenen Teams (aber nicht des momentanen Gegners, der ja - als „you“ - mit diesem Rivalen verglichen wird) ersetzt werden kann:

(5) Are you X in disguise construction

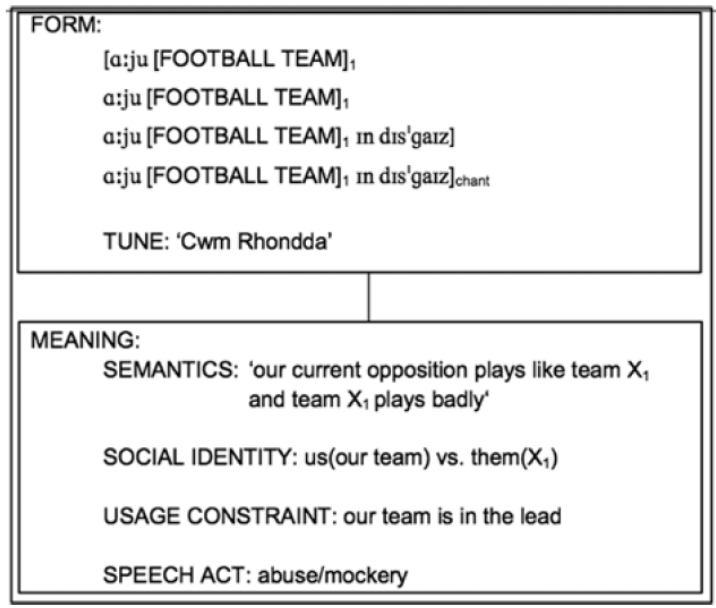

Ein solches Schema ermöglicht es, sowohl die linguistische Struktur mit ihren Variationsmöglichkeiten als auch die - ebenfalls schematisierbare - Semantik und Pragmatik des Chants darzustellen, inklusive der ,Lücken', in denen variiert werden kann, und der semantisch-pragmatischen Vorgaben für diese Variationen. Ein sehr vielversprechender Ansatz also, der allerdings nur für das funktioniert, was er beschreiben kann: kurze, stereotypisierte Gesänge mit Variationen (ein weiteres Beispiel von Hoffmann wäre „You're not V-ing ... any more", in dem V durch sing, ring und ähnliche Verben ausgefüllt wird). Komplexe Fangesänge mit längerem, gänzlich festgelegtem Text - wir werden im Analysekapitel etliche Beispiele davon sehen - können auf diese Weise wohl nicht interpretiert werden.

Doch nun zu den linguistischen Typologien der Fangesänge. Es gibt in der Fachliteratur z. B. die Einteilung von Khodadadi/Gründel (2006, 10-11) ${ }^{9}$ nach Sprechakten und ausgedrückten Gefühlen. Fangesänge teilen sie ein in: (1) unterstützende, (2) solidarische, 
(3) fordernde, (4) euphorische, (5) huldigende, (6) ausgrenzende, (7) diffamierende und (8) irreführende. Außerdem haben sie eine zweite, grundlegendere Unterscheidung, nämlich jene nach (1) „solidarischen“ versus (2) „ausgrenzenden“ Fangesängen (Khodadadi/Gründel 2006, 12-17). ${ }^{10}$ In der vorherigen Liste würden die Typen 1 bis 5 als solidarisch, die Typen 6 bis 8 als ausgrenzend eingestuft werden.

Als linguistisch interessanteste Klassifikation erscheint uns jene von Burkhardt (2009, 181-191), die sich an Sprechakttypen orientiert. Eigentlich handelt es sich um eine Typologie von Sprechgesängen sowie Fahnen- und Spruchbandtexten, ${ }^{11}$ aber man kann sie sehr gut auch auf Fangesänge anwenden. Hier also seine Einteilung, die auf Searles (1982) Sprechaktklassifikation basiert:

1. Direktive Sprechchöre / Fahnen- und Spruchbandtexte [/ Fangesänge]

a) mit stimulierender Intention

b) mit postulierender Intention

c) mit ironisch-ridikülisierender Intention

2. Assertive Sprechchöre / Fahnen- und Spruchbandtexte [/ Fangesänge]

a) mit solidarisierender Intention

b) mit euphorisch-provokativer Intention

c)mit verhöhnender bzw. beleidigender Intention

d)mit ironisch-provokativer Intention

3. Expressive Sprechchöre / Fahnen- und Spruchbandtexte [/ Fangesänge]
a) mit moralisierender Intention
b) mit ausgrenzender Intention
c) mit positiv verstärkender Intention

Burkhardts primäres Einteilungskriterium sind also die Sprechakttypen, aber innerhalb dieser Kategorien fragt er auch immer wieder nach dem participation framework (s. u.). Wir werden es in diesem Beitrag genau umgekehrt machen.

\section{Forschungslücke Romania und neuer Ansatz zur Klassifizierung}

Angesichts einer relativ reichhaltigen Forschung und auch sinnvoller Kategorisierungen fragt man sich, was im Bereich der Fußball-Chants noch zu erforschen wäre. Aus romanistischer und linguistisch-pragmatischer Perspektive nehmen wir zwei Lücken wahr, die noch zu füllen sind: Die erste Lücke ist die Romania; denn wenn auch die deutschen und die englischen Fangesänge ausführlich erforscht und dokumentiert sind, so fehlen doch Studien zu den romanischen Sprachen und Kulturen fast vollständig. Bereits eingangs wurden die zwei Ausnahmen erwähnt, die uns bekannt sind: Minonzio 2014 für Französisch und Mühlbacher 2011 für Spanisch, dazu Harjus 2017 für Portugiesisch, Katalanisch und Spanisch, allerdings über Vereinshymnen. Mühlbacher 2011 arbeitet über Fan-Chants und Hymnen, 
sie folgt weitgehend Struktur und Konzept von Kopiez/Brink 1998 und illustriert das mit spanischen Beispielen. Minonzio 2014 ist eine Art Enzyklopädie über Fußball und Musik, in einem musikwissenschaftlichen Verlag erschienen und sehr lesenswert. Man muss sich allerdings die Artikel über Fan-Chants eigens heraussuchen und findet dort zwar Anekdotisches über einzelne Lieder, aber kein eigentliches Korpus. Für die romanistische Linguistik gibt es somit im Bereich der Fangesänge noch einiges nachzuholen.

Daher soll diese Untersuchung nun den drei ,großen' romanischen Sprachen - Spanisch, Französisch, Italienisch - und den dazugehörigen Fußballkulturen gewidmet sein, und in der neuen Typologie, die hier vorgeschlagen wird, werden alle wichtigen Typen mit romanischen Beispielen illustriert, für die wir jeweils eine deutsche Übersetzung liefern. $\mathrm{Zu}$ erwähnen ist auch, dass es uns hier ausschließlich um die Texte und nicht um die Musik geht, denn unser Ansatz ist ein linguistisch-pragmatischer. Für diese Untersuchung wurde aus der exzellenten Website https://www.fancharts.com (Zugriff 26.04.2018) ein Korpus italienischer, spanischer und französischer Fangesänge zusammengestellt, ${ }^{12}$ das sich für jede Sprache auf drei bedeutende (und rivalisierende) Mannschaften konzentriert, die mit jeweils zehn Items vertreten sind (also insgesamt 90 Gesänge):

- Italien: AC Milan (MI), AC Roma (RO), Juventus (JU),

- Spanien: Real Madrid (RE), Barcelona (BA), Atlético de Madrid (AT),

- Frankreich: Paris Saint-Germain (PS), Olympique Lyonnais (OL), Olympique de Marseille (OM).

Bei der Zusammenstellung wurde vor allem auf Vielfalt geachtet, allerdings mit einer leichten Präferenz für längere Texte, die eine reichere linguistische Ausbeute versprachen, und einer nicht ganz zu leugnenden Schwäche für originelle Beispiele.

Die neue Klassifizierung, die nun hier erprobt werden soll, ist eine Typologie nach Sender-Adressaten-Konstellationen: Wer spricht zu wem? Wer wendet sich an wen? Dafür gibt es verschiedene Fachtermini, z. B. participation framework von Goffman (1979, 11), oder participant constellation von Sacks/Schegloff/Jefferson 1973. ${ }^{13}$ Die Grundbegriffe sollen hier nach Goffman erklärt und auf die Situation beim Fußballmatch übertragen werden. ${ }^{14}$ Goffmans Verdienst in dem berühmten Artikel „Footing“ (1979) ist ja, dass er die starren und zu kurz greifenden Konzepte von „Sprecher/Sender“ und „Hörer/Empfänger“15 aufbricht:

Der Sprecher/Sender teilt sich in folgende Teil-Rollen auf:

- der, der physisch die Worte ausspricht (animator). Beim Fußball-Chant ist das immer die Gruppe der Fans einer der beiden Mannschaften. ${ }^{16}$

- der, der die Worte erfunden, geschrieben hat (author). Wer das bei Fußball-Chants ist, wird in der Fachliteratur ausführlich diskutiert; der chant leader spielt dabei eine besondere Rolle, assistiert von langgedienten Fans aus dem engsten Kreis. 
- der, dessen Meinungen/Ideen wiedergegeben werden (principal). Das ist bei FußballChants wiederum die Gruppe der Fans, der principal ist also identisch mit dem animator; allerdings kommt es vor, dass im Namen von anderen Personen gesprochen wird, das sind dann die ,fiktiven' participation frameworks, für die wir mehrere Beispiele sehen werden.

Der Hörer/Empfänger teilt sich in folgende Rollen auf:

- Angesprochene/r (addressed hearer/s): die Person/en, an die sich der Sprecher wendet. Die Palette ist hier sehr vielfältig, sie reicht von der eigenen Mannschaft über die gegnerische Mannschaft und deren Fans bis zu einzelnen Personen wie einem Spieler oder Trainer oder auch dem Schiedsrichter; auch hier gibt es ,fiktive' Konstellationen, bei denen Abwesende bzw. gedachte Personen angesprochen werden.

- Ratifizierte Hörer (unaddressed hearers): Personen, an die sich der Sprecher zwar nicht wendet, die aber Teilnehmer des gemeinsamen Gesprächs sind. Beim Fußballmatch sind das grundsätzlich einmal sämtliche Beteiligte, das gesamte Publikum (außer den Sängern), beide Mannschaften und ihr Betreuerstab (außer den Teilen davon, die direkt angesprochen werden), die Presse, bzw. auch das Fernsehpublikum, wenn das Match übertragen wird.

- Passanten (bystanders), die sich aufteilen in zwei Gruppen:

- zufällig Mithörende (overhearers): Personen, die zufällig da sind und einen Teil des Gesprochenen mithören;

- Lauscher (eavesdroppers): Personen, die etwas von dem Gespräch mithören wollen, ohne dazu autorisiert zu sein.

Die Gruppe der Passanten ist für die Fußball-Chants nicht so relevant, weil sie ja per definitionem nicht angesprochen sind; man könnte allerdings die Wissenschaftler, die die Gesänge erforschen, in diese Gruppe einordnen.

Eine weitere Kategorie, die bei den Fangesängen relevant ist, wäre die der ,Besprochenen', wenn z. B. im Spiel gegen eine beliebige Mannschaft Chants auftreten, die den Erzrivalen schlechtmachen oder den aktuellen Gegner mit ihm vergleichen. Das wäre dann ein Abwesender, eine Art ,dritte Person', die über die indirekte Adressierung doch in gewisser Weise mit gemeint ist.

Auswertung des Korpus: Verschiedene Sender-Adressaten-Konstellationen mit Beispielen

Wir kommen also jetzt zur Auswertung des Korpus und bringen in der Folge eine Liste aller Sender-Adressaten-Konstellationen, die in den untersuchten Fangesängen vorkommen. In all diesen Konstellationen treten die Fans als Gruppe auf, eben als Chor, während die An- 
gesprochenen oder ,Besprochenen' entweder Gruppen oder auch Einzelpersonen sind; in manchen Liedern spricht ein fiktives Ich, oder man wendet sich an ein fiktives ,Du'. Auch die formal Angesprochenen müssen nicht unbedingt immer präsent sein. (Daher der Titel dieses Beitrags, „Reale und fiktive Sender-Adressaten-Konstellationen“.) Für die meisten Kategorien gibt es übrigens einerseits freundliche, solidarische und andererseits beleidigende, abwertende Varianten; manche Gesänge mischen auch beide Aspekte bzw. sind mehrfach adressiert. Auffällig ist, dass die feindlichen Chants weit weniger kreativ sind als die freundlichen: Sie beschränken sich meist auf nicht sehr originell formulierte Beleidigungen oder Drohungen, wie wir in den Beispielen sehen werden. ${ }^{17}$

\section{ADRESSATEN-VARIATIONEN}

Fans an eine einzelne Person

1. Fans an einen Spieler der eigenen Mannschaft: Diese Chants sind sehr schlicht, es wird nur der Name genannt und wiederholt, sozusagen als Hommage: RE-4 („Ronaldo Ronaldo Ronaldo“) BA-5 („Messi Messi Messi“) BA-7 („Zlatan! Ibrahimovic!“); oder auch als Anfeuerung: JU-5 („Alé Buffon alé alé"); etwas komplexer ist OM-1 („De Marseille à Janeiro Luiz Gustavo (4x) Ouh! Lalalalala lalalalala“). Eine Abwandlung davon wäre Fans an Trainer der eigenen Mannschaft: AT-7 („Oe, oe, oe, oe, ¡Cholo, Cholo!" [= Diego Simeone]); davon gibt es auch eine unzufriedene Variante: RE-8, wenn die Real-Fans Guardiola ironisch attackieren.

2. Fans an einen Spieler (ev. auch Trainer) der gegnerischen Mannschaft ist ebenfalls eine bösartige Variante. Auch diese Chants sind kurz, sie umfassen meist nur den Namen und eine Beleidigung; ein originelleres Beispiel, das sich noch dazu reimt, ist dieses:

\begin{tabular}{|l|l|}
\hline $\begin{array}{l}\text { 1) PS-5 (Fans an einen wohlbeleibten Spieler } \\
\text { der gegnerischen Mannschaft) }\end{array}$ & \\
UN BIG MAC POUR GIGNAC & Ein Big Mac für Gignac \\
\hline Un Big Mac pour Gignac, & Ein Big Mac für Gignac, \\
$\begin{array}{l}\text { Un Big Mac pour Gignac, } \\
\text { Un Big Mac pour Gignac... }\end{array}$ & Ein Big Mac für Gignac, \\
\hline
\end{tabular}

\section{Fans an Schiedsrichter}

Hier wird typischerweise nicht der Name, sondern die Funktionsbezeichnung (árbitro) eingesetzt, kombiniert mit den üblichen, nicht sehr originellen Beleidigungen: AT-5 („Árbitro merengue, merengue hijo de puta“ / „Schiedsrichter, Barcelona-Fan, 
Hurensohn"). Es wird also hier von den Atlético-Fans dem Schiedsrichter vorgeworfen, dass er parteiisch ist.

4. Vielseitig verwendbar ist die allgemeine Beleidigung, deren Adressat sich deiktisch aus der Spielsituation erschließen lässt: RE-9 („Idiota (9 x), hijo de puta“ / „Idiot, Hurensohn").

5. Fans an die eigene Mannschaft, die personifiziert und mit , $\mathrm{Du}^{\prime}$ angesprochen wird: Dieser Variante begegnet man häufig, vor allem, wenn es darum geht, der eigenen Liebe und Treue zum Klub Ausdruck zu verleihen; da wird der Klub personifiziert. Die Beispiele dafür werden in späteren Unterpunkten gegeben werden, wir verweisen dafür u. a. auf Nr. 6, 7, 18, 21, 22, 25.

6. Schließlich gibt es Gesänge, bei denen sich die Fans an ein exemplarisches ,Du'wenden, das keine bestimmte Person ist, sondern ein potentieller Mitstreiter („Komm mit!“) oder irgendjemand aus dem Publikum („Schau, was die tun!“). Wir haben diese Beispiele an anderer Stelle eingeordnet (cf. Bsp. 12 und 20), weil sie auch bezüglich des Senders Besonderheiten aufweisen; aber sie gehören auch hierher als eine originelle Variante eines fiktiven einzelnen Adressaten.

Fans an eine Gruppe

Das ist die Standard-Konstellation der Fan-Chants, aber es gibt davon unzählige Varianten. $\mathrm{Zu}$ bedenken ist, dass immer das gesamte Stadion mit allen Anwesenden (und teilweise auch die TV-Zuschauer) als Publikum, d. h. als ratifizierter Hörer, dabei und also in gewisser Weise als Adressat mit gemeint ist.

7. Die typischste Kategorie ist natürlich Fans an eigene Mannschaft; davon gibt es zahllose Variationen, von denen etliche auch hier illustriert werden sollen. Die Mannschaft wird angefeuert und/oder die Fans erklären ihr ihre Treue - das kann auch mit einem Seitenhieb auf den Erzrivalen verbunden sein.

Beginnen wir mit ein paar kürzeren Beispielen zum Sprechakt „Anfeuern“, der ja oft mit den einfachsten Mitteln auskommt: MI-2 („Alé alé alé, Milan ale“), MI-4 („Alé Milan alé (3 x); i Rossoneri ale““ / „Vorwärts Mailand, vorwärts, Rot-Schwarze“), MI-5 („Siamo qui e cantiamo col cuore, Diavolo [= Spitzname der Mannschaft] vinci per noi, lalala..." / „Wir sind hier und wir singen von Herzen, Teufel, siege für uns..."), RO-1 („Roma Roma Roma Roma, Roma Roma Roma alé, tifosi..." I „Rom... Vorwärts, ihr Fans..."), OL-4 („Allez Lyonnais, allez Lyonnais, allez Lyonnais allez, allez" / „Vorwärts, Lyonnais").

Doch nun zu den längeren Beispielen: Eine wichtige Rolle spielen hier der Imperativ als Modus des Anfeuerns (Bsp. 2), und das Futur, um Treue und Unterstützung zu versprechen, aber auch um zuversichtlich einen Sieg anzukündigen (Bsp. 3): 


\begin{tabular}{|l|l|}
\hline 2) PS-10 (Beispiel für Imperativ) & \\
JOUEZ PARIS SAINT GERMAIN! & Paris Saint Germain, spielt! \\
\hline Allez Paris Saint Germain, & Vorwärts, Paris Saint Germain, \\
Jouez Paris Saint Germain, & Spielt, Paris Saint Germain, \\
Chantez Paris Saint Germain, & Singt, Paris Saint Germain, \\
Allez les Parisiens... & Vorwärts, Pariser... \\
\hline
\end{tabular}

\begin{tabular}{|l|l|}
\hline 3) RE-6 (Beispiel für Futur) & \\
ESTE ESTADIO ESTALLARÁ & Dieses Stadion wird explodieren \\
\hline Ya estamos todos aquí, & Wir sind alle schon da \\
Animando Real Madrid. & Und feuern Real Madrid an. \\
Cuando el Madrid marcará, & Wenn Madrid ein Tor schießt, \\
Este estadio estallará. & Wird das Stadion explodieren. \\
Desde el fondo se alzarán, & Die dann aufstehen werden, \\
Son los hinchas del Real... & Sind die Anhänger von Real... \\
(se repite.... & (bis) \\
\hline
\end{tabular}

Vom Stil her kann es, besonders bei längeren, komplexeren Gesängen, ganz schön pathetisch werden (Bsp. 4) - übrigens so gut wie immer hochsprachlich und mit einer Wortwahl, die vom Ruhm („gloria“, Bsp. 5) bis zur Inbrunst („ferveur“, Bsp. 4) reicht.

\begin{tabular}{|l|l|}
\hline 4) OM-3 (Imperativ, sehr pathetisch) & \\
HISSEZ HAUT LES DRAPEAUX! & Erhebt die Fahnen hoch! \\
\hline Allez l'OM, allez Marseillais, & Vorwärts, OM, vorwärts, Marseiller, \\
Hissez haut les drapeaux! & Erhebt die Fahnen hoch! \\
Tous unis sous les mêmes couleurs, & Alle vereint unter denselben Farben, \\
Le virage reprendra en chour. & Wird die Kurve im Chor antworten. \\
Allez l'OM, allez Marseillais, & Vorwärts, OM, vorwärts, Marseiller, \\
Hissez haut les drapeaux! & Erhebt die Fahnen hoch! \\
Tous unis sous les mêmes couleurs, & Alle vereint unter denselben Farben, \\
Le virage chante avec ferveur... ${ }^{11}$ & Singt die Kurve mit Inbrunst... \\
Forza Milan olé olé olé, & Los, Mailand, olé olé olé, \\
Milan Milan Milan... ${ }^{22}$ & Mailand, Mailand, Mailand... \\
\hline
\end{tabular}




\begin{tabular}{|l|l|}
\hline 5) MI-7 (loben/preisen/anfeuern; pathetisch) & \\
ED I COLORI CHE NOI PORTIAMO & Und die Farben, die wir tragen \\
\hline \multirow{2}{*}{ Ed i colori che noi portiamo } & Und die Farben, die wir tragen, \\
Sono la gloria, & Sind der Ruhm, \\
Sono la gloria. & Sind der Ruhm. \\
Ed i colori che noi portiamo & Und die Farben, die wir tragen, \\
Sono la gloria & Sind der Ruhm \\
Dei Rossone'. & der Rot-Schwarzen. \\
Forza Milan olé, & Los, Mailand, olé, \\
Forza Milan olé, & Los, Mailand, olé, \\
\hline
\end{tabular}

Dieses Beispiel (wie auch unzählige andere) zeigt die Bedeutung der Vereinsfarben, die den Klub - aber auch seine Gegner - jeweils metonymisch repräsentieren. ${ }^{23}$ Der Gesang in Bsp. 5 feuert die eigene Mannschaft nicht nur an, er lobt und preist sie auch. Der pathetische Stil entspricht der geradezu religiösen Verehrung, die die Fans ihrer Mannschaft entgegenbringen. Weiter unten werden wir sehen, dass sogar Drohungen - aus Imponiergehabe heraus - hochsprachlich vorgebracht werden. Nur bei Beleidigungen des Gegners wird die Sprache hemmungslos vulgär. Fangesänge im lokalen Dialekt sind aber tatsächlich die Ausnahme, ${ }^{24}$ in unserem Korpus findet sich genau ein Beispiel dafür (MI-3: „Oh mia bela Madunina...“ / „Oh meine schöne Madunina" ${ }^{\text {"25}}$ ). Immerhin findet sich auch ein Beispiel, in dem die Fans mit ihrer eigenen Mannschaft unzufrieden sind und sie in vulgärer Sprache insultieren: JU-9 („,ci avete rotto il cazzo“ / „ihr geht uns auf den Sack“; wörtlich: „ihr habt uns den Schwanz gebrochen"), und ein einziges (cf. Bsp. 25), wo sie sie mit einem vulgären Ausdruck anfeuern.

Wenn es nicht ganz so läuft, wie die Fans es sich vorstellen, können sie ihrer Mannschaft gegenüber auch ganz schön fordernd werden, sei es mit einer Torforderung (an ein ,Du' gerichtet): JU-1 („Eh facci un gol, Forza Juve facci un gol“ / „Schieß uns ein Tor, Vorwärts Juve, schieß uns ein Tor"), sei es gar mit einer Sieg-Forderung (in WirForm, wahrscheinlich ein inklusives Wir, das Fans und Mannschaft umfasst): JU-10 („Noi vogliamo noi vogliamo, noi vogliamo vincere, vincere, vincere“ / „Wir wollen siegen").

Ein ganz wichtiges Motiv zwischen Fans und Mannschaft ist auch die ewige Treue: BA-10 („Hasta la muerte, el Barcelona hasta la muerte“ / „Bis in den Tod, Barcelona bis in den Tod"). Im folgenden Beispiel werden die Fans als Gruppe einerseits und die Mannschaft als Gruppe andererseits den Einzelpersonen in einer Liebesbeziehung gleichgesetzt (cf. auch die Beispiele 21-28): 


\begin{tabular}{|l|l|}
\hline 6) PS-4 (ewige Treue, wie in Liebesbeziehung) & \\
OÙ TU ES, NOUS SOMMES LÀ & Wo du bist, da sind auch wir \\
\hline Allez Paris, & Vorwärts, Paris, \\
Allez Paris ! Où tu es, & Vorwärts, Paris! Wo du bist, \\
Nous sommes là. & Da sind auch wir. \\
Tu ne seras jamais seule, & Du wirst nie allein sein, \\
Car nous deux, c'est pour la vie... ${ }^{26}$ & Denn das mit uns beiden ist fürs ganze \\
& Leben... \\
\hline
\end{tabular}

Auch ein relativ kurzer Chant kann das Anfeuern, das Ermutigen und die ewige Treue verbinden:

\begin{tabular}{|l|l|}
\hline $\begin{array}{l}\text { 7) MI-10 (anfeuern + ermutigen + ewige } \\
\text { Treue) }\end{array}$ & \\
NON TI LASCEREMO MAI & Nie werden wir dich lassen \\
\hline Alé alé, & Auf auf, \\
Alé Milan alé! & Auf, Mailand, auf! \\
Forza, lotta, & Los, kämpfe, \\
Vincerai. & Du wirst gewinnen. \\
Non ti lasceremo mai... & Nie werden wir dich lassen... \\
(ripeti) & (bis) \\
\hline
\end{tabular}

Mit dem letzten Subtypus von „Fans an eigene Mannschaft“ kommen wir bereits in den Bereich der bösartigen, aggressiven Gesänge, denn es ist eine Aufforderung zur Gewalt gegenüber dem aktuellen Gegner: JU-8 („Uccidete questi bastardi“ / „Tötet diese Bastarde"). Vorher ist aber noch eine kuriose Ausnahme abzuhandeln.

8. Fans an Heimatstadt: In dem folgenden sehr schönen und sehr pathetischen Beispiel verschmelzen die Liebe zur Heimatstadt - die hier personifiziert und mit ,Du“ angesprochen wird - und zur Mannschaft, wobei es sich gut trifft, dass sich „ferveur“ (Inbrunst) mit „cœur“ (Herz) und mit „couleurs“ (Farben) reimt:

\begin{tabular}{|l|l|}
\hline 8) PS-7 (sehr pathetisch) & \\
O VILLE LUMIÈRE & O Stadt des Lichts \\
\hline $\begin{array}{l}\text { O Ville Lumière, } \\
\text { Sens la chaleur }\end{array}$ & $\begin{array}{l}\text { O Stadt des Lichts, } \\
\text { Fühle die Wärme }\end{array}$ \\
\hline
\end{tabular}


De notre cœur !

Vois-tu notre ferveur

Quand nous marchons près de toi,

Dans cette quête.

Chasser l'ennemi,

Enfin, pour que nos couleurs

Brillent encore... ${ }^{28}$

\section{Unseres Herzens!}

Siehst du unsere Inbrunst,

Wenn wir neben dir einhergehen,

In dieser hehren Suche.

Den Feind verjagen,

Endlich, damit unsere Farben

Weiterhin strahlen...

9. Fans an Fans der gegnerischen Mannschaft: Bevor wir zum Typus „Fans an gegnerische Mannschaft" kommen, soll noch ein Lied angeführt werden, in dem die Fans sich dezidiert an die Fans der anderen Mannschaft und nicht an die Mannschaft selbst wenden: nämlich mit dem Vorwurf, die eigenen Lieder zu kopieren und selbst keine Ideen zu haben: AT-1 (,...el lema de los Ultras Sur... es copiar todo lo que hace el Frente, demostrando lo tontos que son..." / „... das Motto der Süd-Ultras ist es, alles zu kopieren, was das Gegenüber macht; so zeigen sie, wie dumm sie sind...").

10. Fans an/über gegnerische Mannschaft (und ihre Fans) (aktueller Gegner)

Nachdem sich die Fan-Gruppen jeweils mit ihrer Mannschaft identifizieren, wird gerade bei diesen Liedern nicht wirklich zwischen Fans und Mannschaft unterschieden, sondern es geht um eine allgemeines, Wir' und ein allgemeines ,Ihr', das als Gegner, ja, als Feind dargestellt wird. Inhaltlich verwandt mit der „Aufforderung an eigene Mannschaft zur Gewalt an Gegnern", aber von der Sender-Adressaten-Konstellation unterschiedlich, ist der wesentlich gängigere Subtypus der Gewaltandrohung an den aktuellen Gegner; von den zahlreichen Beispielen sollen hier nur exemplarisch zwei herausgegriffen werden:

\begin{tabular}{|l|l|}
\hline 9) OM-10 (Gewaltandrohung) & \\
LA CHANSON DES MARSEILLAIS & Das Lied der Marseiller \\
\hline Nous n'offrirons pas de pitié, & Wir werden kein Mitleid haben, \\
Car nous allons vous tuer. & Denn wir werden euch töten. \\
Quand l'OM marquera, & Wenn der OM ein Tor schießt, \\
Tout le stade explosera. & wird das Stadion explodieren. \\
Plus féroce s'élèvera & Und noch gnadenloser erhebt sich \\
La chanson des Marseillais... & Der Gesang der Marseiller... \\
\hline
\end{tabular}

Das vorige Beispiel ist in, Wir-Ihr'-Form gehalten, das folgende ist von der Syntax her ganz unpersönlich, es wird einfach ein Szenario entworfen, aber der indirekte Sprechakt ist natürlich die Drohung, diesen Vorgang, einen Großbrand, herbeizuführen: 


\begin{tabular}{|l|l|}
\hline $\begin{array}{l}\text { 10) JU-6 (implizite Drohung an gegnerische } \\
\text { Mannschaft) }\end{array}$ & \\
FIRENZE IN FIAMME & Florenz steht in Flammen \\
\hline & \\
Un solo grido, & Ein einziger Schrei, \\
Un solo allarme: & Ein einziger Alarm: \\
Firenze in fiamme, & Florenz steht in Flammen, \\
Firenze in fiamme... & Florenz steht in Flammen... \\
\hline
\end{tabular}

Eine weitere Form der indirekten Drohung an den Gegner besteht darin, sich selbst bzw. die eigene Mannschaft als besonders gefährlich und brutal darzustellen; das Beispiel dazu stammt von Mailand und nutzt die Tatsache, dass diese Mannschaft auch als „die Löwen“ bezeichnet wird bzw. es im Stadion eine Kurve namens „Fossa dei Leoni“ gibt. Allerdings ist das schon ein Grenzfall zu „Fans an sich selbst“:

\begin{tabular}{|l|l|}
\hline 11) MI-6 (sich selbst als gefährlich darstellen) & \\
FOSSA LEONI ARMATI & Grube der bewaffneten Löwen \\
\hline Leoni armati, & Als bewaffnete Löwen \\
Stiam marciando, & Marschieren wir, \\
Siam la Fossa dei Leoni, & Wir sind die Löwengrube, \\
Dei leon leon leon, & Die Grube der Löwen, Löwen, \\
Leon leon. & Löwen, Löwen, Löwen. \\
Siam la Fossa dei Leoni, & Wir sind die Löwengrube, \\
Sangue violenza, & Blut, Gewalt, \\
Fossa dei Leoni, & Löwengrube, \\
Milan Milan Milan... & Mailand, Mailand, Mailand... \\
\hline
\end{tabular}

Andere Varianten dessen, was die Fans der gegnerischen Mannschaft singend zu sagen haben, sind natürlich die üblichen, nicht besonders originellen Beleidigungen: RO-3 („E la Lazio merda“ / „Und Lazio Scheisse“), PS-8 („Marseille on t'encule“ / „Marseille, wir ficken dich in den Arsch“), OM-2 („Madrid hijo de puta“ / „Madrid Hurensohn").

11. Fans an/über Erzrivalen-Mannschaft ist verwandt mit dem vorigen Typ, „Fans an Gegner", ist aber in gewisser Weise eine Steigerung davon. Weil die Gegnerschaft zum Erzrivalen so wichtig ist für die Identität der Fans und der eigenen Mannschaft, werden die Songs dieser Kategorie auch dann gesungen, wenn der Erzrivale nicht gerade mit dem aktuellen Gegner identisch ist. D. h., dass sich die Lieder auch an Abwesende richten bzw. Abwesende besingen können. Inhaltlich finden sich Beleidi- 
gungen: BA-4 (Real gegen Barça: „iMadrid! ¡Cabrón! ¡Saluda al campeón!“ / „Madrid! Arschloch! Verneige dich vor dem Sieger!“), AT-8 (Atlético gegen Real: „Madridistas hijos de puta“ / „Madrider, Hurensöhne“); OL-7 (Olympique Lyonnais gegen SaintEtienne: „les Verts, les Verts, on t'encule“ / „Grüne, Grüne, wir ficken dich in den Arsch"), aber auch Gesänge, in denen die Fans proklamieren, wie froh und glücklich sie sind, nicht der Gegner bzw. dessen Fans zu sein: MI-9 („Noi non siamo napoletani“ / „Wir sind keine Napoletaner“) OL-6 („La misère serait de supporter les Verts“ / „Es wäre ein Elend, die Grünen zu unterstützen“); das sind dann vermeintliche Selbstaussagen (daher besteht eine Verwandtschaft zu den „Fans an Mannschaft“- und den „Fans an sich selbst"-Liedern), aber indirekt ist sehr wohl der Erzrivale gemeint.

Weiter oben haben wir das Beispiel von Hoffmann $(2015,278)$ „Are you X in disguise" gesehen, wobei X für den Erzrivalen steht und „you“ den aktuellen Gegner meint; das wäre also eine Kombination aus „Fans an Gegner“ und „Fans über Erzrivalen".

\section{Fans an allgemeine Öffentlichkeit, Publikum}

Das folgende Beispiel richtet sich (in Form eines exemplarischen ,Du', womit das Beispiel auch zu denen gehören könnte, die sich an einen Einzelnen richten, cf. Punkt 6) an die Gesamtheit der Anwesenden, also an das Publikum bzw. eine allgemeine Öffentlichkeit, und es führt (im Zuge des Triumphs eines gewonnenen Spiels) den Gegner vor und verspottet ihn:

12) RE-3 (Verspottung Gegner)
MIRA, MIRA, MIRA
Sacale la foto,
Se van para Gijón con el culo roto...
La, la, la, la, la...
(se repite...) $^{32}$

Schau, schau, schau

Mach ein Foto von ihnen,

Sie fahren nach Gijón mit gebrochenem Arsch...

La, la, la, la, la...

(bis)

13. Fans an sich selbst: In etlichen Liedern besingen die Fans ihre eigene Treue, ihr Zusammengehörigkeitsgefühl. Diese Chants wenden sich schon auch an die Mannschaft, aber nur indirekt. Die Fans bekräftigen, dass sie die Mannschaft unterstützen und dass ihre Unterstützung dieser beim Siegen helfen wird. Tenor ist: ,Wenn wir alle zusammenhalten, wird es gelingen', also eine (direkte oder indirekte) Aufforderung zum Mitmachen, zum Mitsingen: 


\begin{tabular}{|l|l|}
\hline $\begin{array}{l}\text { 13) OM-6 (Aufforderung zum Singen und } \\
\text { Mitmachen) }\end{array}$ & \\
SI NOUS AVONS LA FOI & Wenn wir den Glauben haben \\
\hline $\begin{array}{l}\text { Allez allez Marseillais, } \\
\text { Tous ensemble il faut chanter. } \\
\text { Notre équipe va gagner! } \\
\text { Allez allez Marseillais... }\end{array}$ & $\begin{array}{l}\text { Vorwärts, Marseiller, } \\
\end{array}$ \\
& $\begin{array}{l}\text { Gemeinsam heißt es singen. } \\
\text { Unsere Mannschaft wird gewinnen! } \\
\end{array}$ \\
\end{tabular}

Das Gleiche findet sich auch auf Katalanisch ${ }^{34}$ :

\begin{tabular}{|l|l|}
\hline 14) BA-6 (Aufforderung zum Mitmachen) & \\
I SI TOTS ANIMEN & Und wenn alle mitmachen \\
\hline I si tots animen, & Und wenn alle mitmachen, \\
I si tots animem, & Und wenn wir alle mitmachen, \\
I si tots animem, & Und wenn wir alle mitmachen, \\
Guanyarem, & Werden wir gewinnen, \\
Lo, lo, lo, lo, lo, lo, lo... & Lo, lo, lo, lo, lo, lo, lo... \\
(se repite...) & (bis) \\
\hline
\end{tabular}

Die Kehrseite dieser Kategorie - Fans an sich selbst - sind sehr unfreundliche, bösartige Varianten, die vor allem den gemeinsamen Hass auf die Gegner und Rivalen ausdrücken:

\begin{tabular}{|l|l|}
\hline 15) MI-1 (Hass auf Gegner) & \\
\hline NOI ODIAMO TUTTI LORO & Wir hassen sie alle \\
\hline & \\
Noi odiamo la Juve, & Wir hassen Juventus, \\
Noi odiamo il Verona, & Wir hassen Verona, \\
Noi odiamo la Roma, & Wir hassen Rom, \\
Forza magico Milan... & Vorwärts, magisches Mailand... \\
(ripeti) $^{36}$ & (bis) \\
\hline
\end{tabular}




\section{SENDER-VARIATIONEN}

\section{Reale Untergruppen der Fans}

14. Die Gruppe der Fans singt meist gemeinsam, sie kann sich aber auch in Untergruppen aufsplittern, es kann z. B. eine einzelne Kurve sich explizit als solche äußern (cf. auch oben, Bsp. 11): cf. BA-1 („Hi ha una grada que viu per tu, que no deixa mai d'animar, EEeoo Futbol Club Barcelona“ / „Hier ist eine Tribüne, die für dich lebt, die nie aufhört, dich anzufeuern“) und PS-2 („Debout sur la tribune tous ensemble on va chanter, c'est ce soir tous unis que le virage va crier, PSG allez, PSG allez“ / „Auf der Tribüne stehend werden wir alle gemeinsam singen, heute Abend wird die ganze Kurve vereint schreien, Vorwärts, PSG“). Die originellste Variante ist der Wechselgesang, der Dialog zweier Kurven:

\begin{tabular}{|l|l|}
\hline $\begin{array}{l}\text { 16) PS-6 (Dialog zweier Kurven: } \\
\text { Boulogne und Auteuil) }\end{array}$ & \\
PARIS EST MAGIQUE & Paris ist magisch \\
\hline & \\
Allez Paris, & Vorwärts, Paris, \\
Paris est magique, & Paris ist magisch, \\
Allez Paris, & Vorwärts, Paris, \\
Allez PSG. & Vorwärts, PSG. \\
Allez Paris, & Vorwärts, Paris, \\
Boulogne est magique / Auteuil est & Boulogne ist magisch / Auteuil ist \\
magique, & magisch, \\
Allez Auteuil / Boulogne, & Vorwärts, Auteuil / Boulogne, \\
Allez PSG... ${ }^{37}$ & Vorwärts, PSG... \\
\hline
\end{tabular}

15. Ich-Aussage, Selbstbeweihräucherung der Ultras: Die folgenden beiden Gesänge richten sich zwar an die eigene Mannschaft, aber sie sind vor allem Lobgesänge auf die (in den eigenen Augen) Kerngruppe der Fans, die Ultras:

\begin{tabular}{|l|l|}
\hline $\begin{array}{l}\text { 17) RO-4 (Lobgesang auf Fan-Gruppe } \\
\text { und auf Stadt) }\end{array}$ & \\
GLI ULTRAS DELLA ROMA & Die Ultras von Rom \\
\hline $\begin{array}{l}\text { Gli ultras della Roma siamo noi, } \\
\text { Quelli che combattono per voi, } \\
\text { In un calcio che non ha bandiere, }\end{array}$ & $\begin{array}{l}\text { Die Ultras von Rom sind wir, } \\
\text { Die, die für euch kämpfen, } \\
\text { In einem Spiel ohne Fahnen, }\end{array}$ \\
\hline
\end{tabular}




\begin{tabular}{|l|l|}
\hline Siamo solo noi vecchie maniere. & Nur wir sind nach dem alten Schlag. \\
Non c'è stadio che non ci vedrà, & Kein Stadion wird uns ausweichen, \\
Non c'è gruppo che ci affronterà, & Keine Fangruppe uns standhalten \\
& können. \\
Siam tornati forti più di prima, & Wir sind gestärkt zurückgekehrt, \\
Sul cuore la lupa capitolina, & Auf dem Herzen die Wölfin vom \\
& Kapitol, \\
Oh oh oh oh oh... & Oh oh oh oh oh... \\
\hline
\end{tabular}

18) AT-2 (Lobgesang der Ultras auf sich selbst und ihre Treue)

\section{EN MARCHA VAN LOS ULTRAS}

En marcha van los ultras,

Los ultras del Atleti,

Cantando y animando a nuestro equipo hasta la muerte,

Todos unidos vamos y unidos apoyamos,

Y al conseguir la gloria todos juntos la logramos.

Son rojiblancos nuestros colores, Por los que todos deben sufrir,

Por eso siempre te animaremos,

Te apoyaremos hasta el sin fin,

Lo, lo, lo, lo, lo, lo, lo, lo, lo, lo... ${ }^{39}$
Da kommen die Ultras

Da kommen die Ultras,

Die Ultras von Atlético,

Wir singen und feuern unsere Mannschaft an bis in den Tod,

Wir marschieren vereint und vereint unterstützen wir,

Und wenn es um den Ruhm geht, dann schaffen wir das gemeinsam.

Unsere Farben sind Rot-Weiß,

Für die wir alle leiden müssen,

Daher werden wir dich immer anfeuern,

wir werden dich bis zur Unendlichkeit unterstützen,

Lo, lo, lo, lo, lo, lo, lo, lo, lo, lo...

16. ,Echte' Fans an lauwarm Mitmachende: Die bereits weiter oben unter Punkt 13 (Bsp. 13 und 14) illustrierte Variante der Aufforderung zum Mitmachen hat eine aggressive Variante, bei der die selbst ernannten ,echten' Fans die vielleicht weniger radikalen ausgrenzen (diesen Text gibt es übrigens in etlichen Variationen, er ist sehr verbreitet; allein in unserem Korpus finden sich drei Exemplare davon $)^{40}$ :

19) JU-3 (Beleidigung der nur lawwarm Mitmachenden)

CHI NON CANTA Wer nicht (mit)singt 


\begin{tabular}{|l|l|}
\hline E chi non canta è un figlio di puttana, & $\begin{array}{l}\text { Und wer nicht singt, ist ein } \\
\text { Eurensohn, }\end{array}$ \\
E chi non canta va a vedere il Toro, & $\begin{array}{l}\text { Und wer nicht singt, soll zum Match } \\
\text { von Turin gehen, }\end{array}$ \\
E chi non canta resta a casa, & Und wer nicht singt, bleibt zu Hause, \\
Che cazzo viene a fare qua? & Was zum Teufel hat er denn hier zu \\
& suchen? \\
Alé alé, alé alé... ${ }^{41}$ & Gemma gemma, gemma gemma... \\
\hline
\end{tabular}

17. (Süd-)Ultras an potentielle Mitstreiter: Die folgende Aufforderung zum Mitmachen ist an ein fiktives ,Du' (cf. Punkt 6), einen vielleicht weniger aktiven Fan, gerichtet. Sie passt als ,freundlichere' Variante zum vorigen Typus. Freundlicher ist sie allerdings nur den weniger radikalen Fans gegenüber, während der Erzrivale als Schwein bezeichnet wird, das getötet werden soll:

\begin{tabular}{|l|l|}
\hline $\begin{array}{l}\text { 20) RE-2 (Aufforderung zum Mitmachen bei } \\
\text { der Gewalt) }\end{array}$ & \\
VENTE CON LOS ULTRAS-SUR & Komm mit mit den Süd-Ultras \\
\hline $\begin{array}{l}\text { Vente con los Ultras-Sur, } \\
\text { Que vamos a Barcelona. }\end{array}$ & $\begin{array}{l}\text { Komm mit mit den Süd-Ultras, } \\
\text { Wir fahren nach Barcelona. }\end{array}$ \\
$\begin{array}{l}\text { Hay que quemar el Nou Camp, } \\
\text { Con cien mil bastardos dentro. }\end{array}$ & $\begin{array}{l}\text { Mit hunderttausend Bastarden drin. } \\
\text { Que es la forma más barata, } \\
\text { De acabar con tanto cerdo... }{ }^{42}\end{array}$ \\
& $\begin{array}{l}\text { Denn das ist die billigste Art, } \\
\text { So vielen Schweinen den Garaus } \\
\text { zu machen... }\end{array}$ \\
\hline
\end{tabular}

Fiktive einzelne Fans als Sender

18. Ein (exemplarischer) Fan (,ich') erklärt dem Klub (,du') seine Gefühle: Liebe und Treue - aber auch Hass gehört in diesen bekenntnishaften Chants dazu.

Dass die Beziehung des Fans zu seinem Klub (neben einem religiösen Bezug) mit einer Liebesbeziehung gleichgesetzt wird, verwundert ja nicht wirklich. Interessant ist allerdings, dass die Chants, die diese Metapher ausspinnen, alle in Ich-Form gehalten sind und sich dementsprechend auch an ein ,Du' wenden (cf. oben, Punkt 5). Hier gibt es kein, Wir' mehr, die Beziehung ist intim, von Individuum zu Individuum. Nachdem die Lieder von einem vieltausendstimmigen Chor gesungen werden, ist dieses ,Ich' ein fiktiver Sender; animator und principal fallen auseinander, weil der principal eine imaginäre Person ist. Ebenso wird der Klub personifiziert und ist damit 
ein fiktiver Empfänger. Vielleicht ist es aber auch so, dass diese Art von Liedern jeder einzelne Fan ganz für sich singt, dann ist es ein Chor einzelner verliebter Herzen, die allerdings das angesungene Objekt der Liebe teilen. Motive sind die Treue: OL-8 („Toute l'Europe je traverserai“ / „Ganz Europa werde ich durchmessen“) und natürlich die Liebe, wie in den folgenden Liedern:

\begin{tabular}{|c|c|}
\hline \multirow{2}{*}{$\begin{array}{l}\text { 21) AT-4 (Treue und Religion) } \\
\text { YO NACÍ ENAMORADO DEL ATLETI } \\
\text { DE MADRID }\end{array}$} & \multirow[b]{2}{*}{$\begin{array}{l}\text { Ich war schon in Atlético Madrid ver- } \\
\text { liebt, als ich geboren wurde }\end{array}$} \\
\hline & \\
\hline Y yo nací enamorado del Atleti de Madrid, & $\begin{array}{l}\text { Ich war schon in Atlético Madrid } \\
\text { verliebt, als ich geboren wurde, }\end{array}$ \\
\hline En las canchas donde tanto yo sufrí, & $\begin{array}{l}\text { Auf den Spielfeldern, wo ich so viel } \\
\text { gelitten habe, }\end{array}$ \\
\hline Enamorado de ti, & Verliebt in dich. \\
\hline Tus rayas son & Deine Streifen sind \\
\hline Rojas y blancas que llevo en el corazón, & $\begin{array}{l}\text { Rot und weiß, und ich trage sie im } \\
\text { Herzen, }\end{array}$ \\
\hline $\begin{array}{l}\text { Hacen que sean como una religión, } \\
\text { Cantemos el alirón... } \\
(\text { se repite.... })^{43}\end{array}$ & $\begin{array}{l}\text { Sie sind wie eine Religion, } \\
\text { Singen wir den Siegesgesang... } \\
\text { (bis) }\end{array}$ \\
\hline
\end{tabular}

\begin{tabular}{|l|l}
\hline $\begin{array}{l}\text { 22) AT-10 (Ein Fan erzählt, wie er sich } \\
\text { verliebt hat) }\end{array}$ & \\
MAS ALLÁ DE LA CURIOSIDAD & Über die Neugier hinaus \\
\hline Mas allá de la curiosidad & Über die Neugier hinaus \\
Surgió un amor, & Entstand eine Liebe, \\
La primera vez & Als ich das erste Mal das \\
Que yo entré en el Calderón. & Calderón-Stadion betrat. \\
$\begin{array}{l}\text { De tus colores me enamoré, } \\
\text { Sentí pasión, }\end{array}$ & Ich verliebte mich in deine Farben, \\
Dentro de mi, & Ich empfand Leidenschaft, \\
Late muy fuerte mi corazón... ${ }^{44}$ & Und in mir drin \\
\hline
\end{tabular}

Eine originelle Variante dieser Bekenntnislieder ist die folgende, wo es nicht um Liebe, sondern um Sucht, um Besessenheit geht: 


\begin{tabular}{|l|l|}
\hline 23) OL-2 (Fankult als Sucht) & \\
COMME UNE MALADIE & Wie eine Krankheit \\
\hline Ma drogue c'est l'Olympique Lyonnais, & Meine Droge, das ist der Olympique \\
& Lyonnais, \\
Je ne pourrai jamais m'en passer. & Ich werde nie ohne ihn auskommen. \\
C'est comme une maladie, & Es ist wie eine Krankheit, \\
Je n'en serai jamais guéri. & Nie werde ich davon geheilt werden. \\
Lololololololo, & Lololololololo, \\
Lolololololo, & Lolololololo, \\
Heyhey, ho, & Heyhey, ho, \\
Heyhey, ho... ${ }^{45}$ & Heyhey, ho... \\
\hline
\end{tabular}

Ein Topos sind auch Lieder wie die folgenden, in denen ein exemplarischer Fan erklärt: Mir ist egal, was die anderen denken ${ }^{46}$, ich liebe diesen meinen Verein und bleibe ihm treu. Mit den anderen, die zitiert werden, wird übrigens in einer Art Polyphonie ein weiterer Sprecher indirekt in Szene gesetzt:

\begin{tabular}{|c|c|}
\hline \multirow{2}{*}{$\begin{array}{l}\text { 24) RO-6 (egal, was andere sagen) } \\
\text { DICONO VAI ALLO STADIO }\end{array}$} & \\
\hline & Sie sagen, du gehst ins Stadion \\
\hline Dicono vai allo stadio sei un deficiente! & $\begin{array}{l}\text { Sie sagen, du gehst ins Stadion, du bist } \\
\text { ein Idiot! }\end{array}$ \\
\hline Dicono l'AS Roma non vince niente, & Sie sagen, der AS Rom gewinnt nie, \\
\hline Ma io me ne frego, resto al mio posto, & $\begin{array}{l}\text { Aber mir ist das egal, ich bleibe an mei- } \\
\text { nem Platz, }\end{array}$ \\
\hline Io me ne frego, so' giallorosso! ${ }^{47}$ & Mir ist das egal, ich bin gelb-rot! \\
\hline
\end{tabular}

\begin{tabular}{|l|l|}
\hline 25) RE-7 (anfeuern + egal, was andere sagen) & \\
\hline VAMOS REAL MADRID & Vorwärts, Real Madrid \\
\hline Vamos Real Madrid te sigo a todas partes, & $\begin{array}{l}\text { Vorwärts, Real Madrid, ich folge dir } \\
\text { überallhin, }\end{array}$ \\
Yo te quiero, & $\begin{array}{l}\text { Ich liebe dich, } \\
\text { Vamos a dar la vuelta a todo el mundo. }\end{array}$ \\
¡Hay que ponerle un poco más de huevos* \\
Más de huevos*! & $\begin{array}{l}\text { Es braucht ein bisschen mehr Rückgrat* } \\
\text { Y eso que dice la gente, }\end{array}$ \\
\hline
\end{tabular}


Que somos borrachos, vagos, delincuentes,

No le hago caso,

Voy a todos los lados,

Yo soy del Madrid,

Soy un descontrolado,

Lo, lo, lo, lo...48
Dass wir Säufer sind, Feiglinge,

Verbrecher,

Das nehm' ich mir nicht zu Herzen,

Ich fahre überallhin,

Ich gehöre zu Madrid,

Ich bin außer Rand und Band,

Lo, lo, lo, lo...

*huevos: Eier (=Hoden)

Die Konstellation mit dem einzelnen, fiktiven exemplarischen Fan, der ein Bekenntnis ablegt, hat auch eine feindselige Variante, bei der das ,Ich' dem oder den Erzrivalen ewigen Hass schwört, ja, sogar wünscht, ihn/sie zu töten:

\begin{tabular}{|l|l|}
\hline 26) JU-7 (Fan-Bekenntnis: Hass) & \\
FINCHÉ VIVRÒ & Solange ich lebe \\
\hline Finché vivrò, odierò, & $\begin{array}{l}\text { Solange ich lebe, werde ich sie hassen, } \\
\text { Viola e Granata, }\end{array}$ \\
$\begin{array}{l}\text { Odierò, } \\
\text { La polizia }\end{array}$ & $\begin{array}{l}\text { Werde ich hassen, } \\
\text { Per sempre odierò... }{ }^{49}\end{array}$ \\
& $\begin{array}{l}\text { Die Polizei } \\
\text { Werde ich ewig hassen... }\end{array}$ \\
& $\quad *$ die Farben von Fiorentina und Torino \\
\hline
\end{tabular}

27) BA-2 (Fan-Bekenntnis: Feindschaft, töten)

SI ALGUNA VEZ

Wenn ich einmal

Si alguna vez,

Si alguna vez,

He de matar,

He de matar,

Que sea un perico perico de Sarriá,

¡Barça!...

(se repite...) $)^{50}$
Wenn ich einmal,

Wenn ich einmal

Töten sollte,

Töten sollte,

Möge es ein Perico* aus Sarriá** sein,

Barça!...

(bis)

${ }^{*}$ perico $=$ Fan von RCD Español Barcelona

** Sarrià $=$ Stadtteil, wo früher das Stadion von Español stand 
19. Eine Sondervariante des Treuemotivs bilden Lieder, in denen ein exemplarischer Fan sich an die (zu Hause gebliebene) Partnerin oder Mutter wendet und rechtfertigt bzw. erklärt, warum der Sonntag der eigenen Mannschaft gehört. Hier wird also eine fiktive Adressatin in Szene gesetzt:

\begin{tabular}{|l|l|}
\hline $\begin{array}{l}\text { 28) OL-5 (Fan erklärt Mutter die Liebe zu } \\
\text { seiner Mannschaft) }\end{array}$ & \\
MAMAN & Mutti \\
\hline Oh la maman maman, & Oh la Mutti Mutti, \\
Sais-tu pourquoi & weißt du, warum \\
Mon cœur est rouge et bleu? & mein Herz rot und blau ist? \\
J'ai vu jouer les Lyonnais, & Ich habe Lyon spielen gesehen, \\
Et c'est pour ça que mon cœur est rouge et & und deshalb ist mein Herz rot und \\
bleu... & blau... \\
(répéter...) & (bis) \\
\hline
\end{tabular}

Fiktive andere Personen als Sender

20. In meinem wahrscheinlich originellsten Beispiel spricht die (fiktive) zu Hause gebliebene Partnerin und macht dem (fiktiven einzelnen) Fan Vorwürfe, was indirekt dessen Liebe zum Klub anklingen lässt:

\begin{tabular}{|l|l|}
\hline $\begin{array}{l}\text { 29) RO-7 (Es spricht die vernachlässigte } \\
\text { Partnerin des Fans) }\end{array}$ & \\
DA SOLA & Allein \\
\hline & \\
Perché, perché, & Warum, warum, \\
La domenica mi lasci sempre sola, & Lässt du mich am Sonntag immer allein, \\
Per andare a vedere la partita, & Um beim Spiel von Rom \\
Della Roma...52 & Dabei zu sein... \\
\hline
\end{tabular}

Man muss sich vorstellen, dass wahrscheinlich ein erheblicher Teil der Fans genau diese Szene vor der Abfahrt zu Hause erlebt hat. Hier stärken sie sich gegenseitig den Rücken und solidarisieren sich augenzwinkernd als Leidtragende dieses Vorwurfs. 


\section{Konklusion}

Fußball-Fangesänge haben den Ruf, eher einfach, ja, regelrecht primitiv zu sein: Sie übernehmen bekannte Melodien und vereinfachen diese wenn notwendig noch; die Mannschaften schauen sich gegenseitig die Lieder ab, oft mit ganz ähnlichem Text. ${ }^{53}$ Man würde fast glauben, das muss eben so sein, weil man Gruppen von Tausenden, ja Zigtausenden Leuten nicht komplexe Melodien und Texte singen lassen kann, und weil der Zweck nicht künstlerische Erbauung, sondern Anfeuern, Emotionsausdruck und Zusammengehörigkeitsgefühl ist. Diese Untersuchung, die ausschließlich von den (französischen, italienischen und spanischen) Texten ausgegangen ist, hat aber gezeigt, dass die immer ähnlichen Themen in einer großen Vielzahl von Ausdrucksformen behandelt werden. Wir haben die Einteilung nach Sprechakten und Emotionen beiseitegelassen und die Gesänge nach dem eingeteilt, was Goffman als participation framework und die Konversationsanalyse als participant constellation bezeichnet, also: Wer spricht zu wem? Wer geriert sich als Sender (oder fiktiver Sender)? Wer ist der Adressat (bzw. der indirekte Adressat)? Dabei ist eine Fülle an verschiedenen Partner-Konstellationen ans Licht getreten, die teilweise sehr originell und sehr komplex sind und die Kreativität und den Humor der singenden Fangemeinschaften bezeugen. Es hat sich gezeigt, dass sich der aus der Fangemeinde eines Klubs bestehende Chor in den Chants an die verschiedensten Adressaten wendet: an die eigene Mannschaft, um sie anzufeuern, an die Gegner (Mannschaft und/oder Fans), um sie zu schmähen, vorzuführen ${ }^{54}$ oder zu bedrohen, aber auch (selbst in Abwesenheit) an den Erzrivalen und seine Fans. Und nicht zuletzt wenden sich die Fan-Chöre immer wieder auch an sich selbst, um ihren Zusammenhalt, ihre Liebe und Treue zur Mannschaft und ihren gemeinsamen Hass zu erklären. Ein Beispiel ist aber auch an die Heimatstadt adressiert, die metonymisch für die geliebte Mannschaft steht. Wenn Einzelpersonen angesprochen werden, dann handelt es sich um den Schiedsrichter, um Spieler oder Trainer der eigenen und der gegnerischen Mannschaft. In der fiktiven Variante geht es um die Mannschaft selbst, die mit „Du“ angesprochen und damit personifiziert wird, oder auch um fiktive potentielle Mitstreiter, die zum Mitmachen, Mit-Lieben, Mit-Hassen aufgefordert werden.

Nicht minder vielfältig erweisen sich die Sender-Konstellationen, denn die Identifikation des Chors mit der im Stadion anwesenden Fan-Gemeinde einer Mannschaft stellt nur eine von zahlreichen möglichen Sender-Varianten dar. Da gibt es z. B. Äußerungen von Untergruppen wie etwa von zwei Stadion-Kurven im Wechselgesang, und ganz speziell von der Untergruppe der Ultras, die sich als die einzig wahren Fans begreifen und sich auch immer wieder selbst beweihräuchern. Für diese sind dann die weniger radikalen Fans eine Gruppe, die zum Mitmachen aufgefordert wird. Auffällig sind bei den Sendern allerdings die fiktiven Typen, wie z. B. die daheimgebliebene Partnerin, die dem treu die Mannschaft begleitenden Fan mit Unverständnis, ja, mit Vorwürfen begegnet; und ganz besonders der fiktive einzelne Fan, der der zur Geliebten mutierten Mannschaft seine Liebe erklärt und seine ewige Treue schwört. 
In all diesen Konstellationen wird in mannigfaltiger Weise Zugehörigkeit inszeniert und produziert, das Zusammengehörigkeitsgefühl einer Wir-Gruppe, die durch gemeinsame Liebesgefühle gegenüber der eigenen Mannschaft und Hassgefühle gegenüber den Gegnern zusammengeschweißt wird. Auffällig ist auch, dass dem individuellen Fan und seinen Schwierigkeiten - z. B. zu Hause, wenn er Vorwürfen ausgesetzt ist - gezielt Rechnung getragen und ihm der Rücken gestärkt wird, um ihn noch stärker in die Gruppe hereinzuholen.

Zum Abschluss sei hier ein besonders originelles Beispiel des zuletzt besprochenen Typs zitiert, nämlich „Bekenntnis eines einzelnen Fans = Liebeserklärung an seine Mannschaft". In dem folgenden - nicht ganz ernst gemeinten - Lied wird die eigene Mannschaft (Marseille) einer besonders typischen heimischen Spirituose, dem „Pastis 51“, gleichgesetzt: ein emblematisches Symbol der Zugehörigkeit. ${ }^{55}$ Die Alternative, die in der zweiten Strophe anklingt, ist übrigens ein ganz ähnliches Getränk derselben Firma:

\begin{tabular}{|l|l|}
\hline $\begin{array}{l}\text { 30) OM-4 (Treue + Identifikation mit lokalen } \\
\text { Spirituosen) }\end{array}$ & \\
51, JE T'AIME! & 51 , ich liebe dich! \\
\hline 51, je t'aime! & 51, ich liebe dich! \\
J'en boirais des tonneaux & Ich würde ganze Fässer trinken, \\
À me rouler par terre & Bis ich mich am Boden rolle, \\
Dans tous les caniveaux. & In allen Rinnsalen. \\
& \\
Et si tu m'abandonnes & Und wenn du mich fallen lässt, \\
Alors je m'empoisonne & Dann vergifte ich mich \\
Avec une bonbonne & Mit einer großen Flasche \\
D'un Ricard bien jaune... & Voll schön gelbem Ricard... \\
\hline
\end{tabular}

\section{Endnoten}

1 Eva Lavric ist Professorin für romanische Sprachwissenschaft am Institut für Romanistik der Universität Innsbruck.

2 Aus musikwissenschaftlicher Perspektive: Kopiez/Brink 1998, Kopiez 2002, Brunner 2009; aus ethnologischer: Rühlemann 1996, Bromberger 1995 und 1998, Soder 2003, Schmidt-Lauber 2009; aus kulturwissenschaftlicher: Luhrs 2008, Mühlbacher 2011; aus linguistischer: Khodadadi/ Gründel 2006, Schiering 2008, Burkhardt 2009, Hoffmann 2015, Bastian/Koch 2015.

3 Aus Gründen der Kürze sowie der Abwechslung wird hier auch häufig der englische Ausdruck Chants verwendet.

$4 \mathrm{Zu}$ Spanisch, Katalanisch und Portugiesisch gibt es außerdem Harjus 2017, allerdings über Vereinshymnen. 
5 Der Vergleich stammt von Kopiez/Brink (1998, 227); er findet sich auch in Rosentritt (2001).

6 Man erinnere sich dabei an Tajfels Theorie vom Antagonismus zwischen Ingroup und Outgroup (cf. Tajfel 1974).

7 Hoffmann $(2015,279)$ spricht vom „mocking and verbal abuse of the (present and virtual) opposition".

8 Allerdings gibt es auch Beispiele wie das von Antoine Griezmann, der von den Fans seines langjährigen Klubs Atlético Madrid mit Pfiffen und „Griezmann, stirb“-Gesängen heftig attackiert wurde, weil er ausgerechnet beim Erzrivalen FC Barcelona angeheuert hatte (cf. Griezmann 2019). Dass Atlético nun wegen dieses Ausrutschers seiner Fans eine Beschwerde seitens der spanischen Liga droht, zeigt, dass derartige Attacken die üblichen Fairness-Verhaltenscodes sprengen und bestätigt so indirekt die Existenz dieser Codes.

9 Die Einteilung bezieht sich auf Fangesänge und Vereinslieder, wobei die letzten drei Kategorien nur bei Fangesängen vorkommen. Wir interessieren uns hier nur für Fangesänge.

10 Am Ende des Artikels bringen die Autoren außerdem eine Analyse zweier deutscher Vereinslieder.

11 Das liegt daran, dass es in dem Sammelband Flickflack, Foul und Tsukahara, den Burkhardt/ Schlobinski 2009 herausgegeben haben, einen eigenen Artikel (Brunner 2009) gibt, der unmittelbar nach Burkhardts Beitrag steht und der seinerseits die Fangesänge behandelt. Brunner ist allerdings ein Musikwissenschaftler und stark von Kopiez/Brink 1998 inspiriert.

12 Dabei wurden die dort gegebenen Transkriptionen grundsätzlich übernommen, allerdings bezüglich Satzzeichen, Groß- und Kleinschreibung etc. soweit notwendig geglättet.

13 Für eine gut verständliche Erklärung cf. Auer (1984, 32-33).

14 Ganz ähnlich erklärt das Hoffmann $(2015,279)$. Er spricht von audience design und führt den Begriff auf Bell (1984) zurück. Die Kategorien, die er unterscheidet, finden sich allerdings so bereits in Goffman (1979, 7-11).

15 Aus Gründen der Lesbarkeit verzichten wir auf das Gendern.

16 Bei Wechselgesängen kann auch der chant leader mit der Gruppe alternieren; bzw. kommt es auch zum Alternieren zwischen verschiedenen Fan-Gruppen (s. u.).

17 Kurzgesänge oder Ausschnitte werden im Fließtext in Klammern eingefügt. Nur für längere Chants, die vollständig zitiert werden, wird ein eigenes Beispielsformat mit Nummerierung eingeführt; als Quellenangabe fungiert der Name des Vereins und eine Zahl von 1 bis 10, die eine Art laufende Nummer ist. RO-3 bezeichnet also den dritten der zehn für Rom ausgewählten Gesänge.

18 https://www.fanchants.com/football-songs/psg-chants/un-big-mac-pour-gignac/ (Zugriff26.04.2018).

19 https://www.fanchants.com/football-songs/psg-chants/play-2/ (Zugriff 26.04.2018).

20 https://www.fanchants.com/football-songs/real-madrid-chants/madrid-chant/ (Zugriff 26.04.2018).

21 https://www.fanchants.com/football-songs/marseille-chants/hissez-haut-les-drapeaux/ (Zugriff 26.04.2018).

22 https://www.fanchants.com/football-songs/ac-milan-chants/edicolori/ (Zugriff 26.04.2018).

23 Eine ähnliche, aber wesentlich geringere Rolle spielt auch das Heimstadion der Mannschaft, es steht ebenfalls oft metonymisch für den Klub (cf. Bsp. 20 und 22). 
24 Schiering 2008 befasst sich mit der Frage, warum trotz lokaler Identität die Fans der Fußballklubs nicht im lokalen Dialekt singen. Im Wesentlichen geht es darum, dass es sich um ein öffentliches Singen handelt und dass Fußball eine zu ernste Sache ist, um ihn locker im Dialekt abzuhandeln.

25 „Der [sic] Kanzone ,Oh mia bela Madunina“ oder ,La Madunina' ist die inoffizielle Hymne der Stadt Mailand. Der Titel bezieht sich auf la Madonnina (,kleine Madonna', im Mailänder Dialekt ,Madunina'), die goldene Madonnenstatue auf dem Turm des Mailänder Doms, die weitherum in der Stadt sichtbar ist. ,La Madonnina' ist im lombardischen Dialekt verfasst, für den es keine festgelegte Schreibweise gibt. Das Lied ist unter den Milanesi sehr populär, man kann es als Klingelton für das Handy bekommen, und es gehört zu den Fan-Liedern sowohl der Curva Sud des Fußballklubs AC Mailand, beispielsweise der Brigate Rossonere, als auch der Curva Nord des Fußballklubs Inter Mailand, die den Text sogar auf ihren Homepages verbreiten.“ (https://de.wikipedia.org/wiki/Oh_mia_bela_Madunina, Zugriff 29.11.2019). Danke an Angelo Pagliardini für den Hinweis!

26 https://www.fanchants.com/football-songs/psg-chants/come-on-paris/ (Zugriff 26.04.2018).

27 https://www.fanchants.com/football-songs/ac-milan-chants/we-wont-leave-you-3/ (Zugriff 26.04.2018).

28 https://www.fanchants.com/football-songs/psg-chants/oh-ville-lumiere/ (Zugriff 26.04.2018).

29 https://www.fanchants.com/football-songs/marseille-chants/the-song-of-marseillais/ (Zugriff 26.04.2018).

30 https://www.fanchants.com/football-songs/juventus-chants/florence/ (Zugriff 26.04.2018).

31 https://www.fanchants.com/football-songs/ac-milan-chants/fossa/ (Zugriff 26.04.2018).

32 https://www.fanchants.com/football-songs/real-madrid-chants/look/ (Zugriff 26.04.2018).

33 https://www.fanchants.com/football-songs/marseille-chants/if-we-have-faith/ (Zugriff 26.04.2018).

34 Die Fan-Chants von Barcelona sind allerdings nicht durchgehend auf Katalanisch, sondern nur gelegentlich; ob sich das in letzter Zeit geändert hat, wäre interessant zu beobachten.

35 https://www.fanchants.com/football-songs/barcelona-chants/i-si-tots-animen/ (Zugriff26.04.2018).

36 https://www.fanchants.com/football-songs/ac-milan-chants/we-hate-11/ (Zugriff 26.04.2018).

37 https://www.fanchants.com/football-songs/psg-chants/paris-est-magique/ (Zugriff 26.04.2018).

38 https://www.fanchants.com/football-songs/roma-chants/only-us-2/ (Zugriff 26.04.2018).

39 https://www.fanchants.com/football-songs/atletico-madrid-chants/en-marcha-van-los-ultras/ (Zugriff 26.04.2018).

40 Dieser Chant - in englischer Fassung - gehört übrigens auch zu jenen, die Hoffmann 2015 als "Constructions" analysiert.

41 https://www.fanchants.com/football-songs/juventus-chants/home-7/ (Zugriff 26.04.2018).

42 https://www.fanchants.com/football-songs/real-madrid-chants/vente-con-los/ (Zugriff 26.04.2018).

43 https://www.fanchants.com/football-songs/atletico-madrid-chants/15/ (Zugriff 26.04.2018).

44 https:/www.fanchants.com/football-songs/atletico-madrid-chants/15-4/ (Zugriff 26.04.2018).

45 https://www.fanchants.com/football-songs/lyon-chants/us-2/ (Zugriff 26.04.2018).

46 Eine Anspielung auf den schlechten Ruf der Fußballfans und die Kritik an ihnen, cf. auch unten, Bsp. 29 und 30. 
47 https://www.fanchants.com/football-songs/roma-chants/dicono-vai-allo-stadio/ (Zugriff 26.04.2018).

48 https://www.fanchants.com/football-songs/real-madrid-chants/come-real-madrid-i-follow-youeverywhere/ (Zugriff 26.04.2018).

49 https://www.fanchants.com/football-songs/juventus-chants/always-14/ (Zugriff 26.04.2018).

50 https://www.fanchants.com/football-songs/barcelona-chants/sometime/ (Zugriff 26.04.2018).

51 https://www.fanchants.com/football-songs/lyon-chants/mamma/ (Zugriff 26.04.2018).

52 https://www.fanchants.com/football-songs/roma-chants/all-alone/ (Zugriff 26.04.2018). Es handelt sich um ein Lied von Rita Pavone aus den 60er Jahren (1962), das textlich adaptiert und vereinfacht wurde. Dank an Gerhild Fuchs für diesen Hinweis!

53 Was sie sich gegenseitig dann auch wieder vorwerfen: cf. das Beispiel AT-1 unter Punkt 9. im Analysekapitel).

54 In diesem Fall ist der Adressat ein allgemeines Publikum.

55 Insofern würde dieses Beispiel eigentlich auch gut zu Bsp. 8 unter Punkt 8 passen, in dem nicht die Mannschaft, sondern an ihrer Stelle die Stadt, als metonymische Vertreterin, angesprochen wird.

56 https://www.fanchants.com/football-songs/marseille-chants/51-i-love-you/ (Zugriff 26.04.2018).

\section{Bibliographie}

Askin, Ridvan / Diederich, Catherine / Bieri, Aline (Hg.): The Aesthetics, Poetics, and Rhetoric of Soccer. London / New York: Routledge, 2018.

Auer, Peter: Bilingual Conversation. Amsterdam/Philadelphia: Benjamins, 1984.

Bastian, Sabine / Koch, Florian: „Le dénigrement de l'autre par le biais de la langue. Une étude pilote dans le domaine du foot en France“. In: Revue d'Études Françaises 20 (2015), 23-36.

Bell, Allen: „Language Style as Audience Design“. In: Language in Society 13 (1984), 145-204.

Belliger, Andréa / Krieger, David J. (Hg.): Ritualtheorien. Ein einführendes Handbuch. Opladen/ Wiesbaden: Westdeutscher Verlag, 1998.

Bromberger, Christian: „Football as World-View and as Ritual“. In: French Cultural Studies 6 (1995), 293-311.

Bromberger, Christian: „Fussball als Weltsicht und als Ritual“. In: Belliger, Andréa / Krieger, David J. (Hg.): Ritualtheorien. Ein einführendes Handbuch. Opladen/Wiesbaden: Westdeutscher Verlag, 1998, 285-301.

Brunner, Georg: „Fangesänge im Fußballstadion“. In: Burkhardt, Armin / Schlobinski, Peter (Hg.): Flickflack, Foul und Tsukahara. Der Sport und seine Sprache. Mannheim u. a.: Dudenverlag, 2009, 194-210.

Burkhardt, Armin: „Der zwölfte Mann. Fankommunikation im Fußballstadion“. In: Burkhardt, Armin / Schlobinski, Peter (Hg.): Flickflack, Foul und Tsukahara. Der Sport und seine Sprache. Mannheim u. a.: Dudenverlag, 2009, 175-193.

Burkhardt, Armin / Schlobinski, Peter (Hg.): Flickflack, Foul und Tsukahara. Der Sport und seine Sprache. Mannheim u. a.: Dudenverlag, 2009. 
Goffman, Erving: „Footing“. In: Semiotica 25,1-2 (1979), 1-29.

[Griezmann]: „Griezmann, stirb“: Atlético-Fans bereiten Barcelona-Star bei Rückkehr üblen Empfang. Sportbuzzer, 02.12.2019, https://www.sportbuzzer.de/artikel/atletico-madrid-antoinegriezmann-fans-fc-barcelona-ruckkehr-beleidigung-eklat/ (Zugriff 04.12.2019).

Harjus, Jannis: „El corazón de la afición está contigo’. Un acercamiento lingüístico-discursivo a los himnos oficiales de fútbol catalanófonos, lusófonos e hispanófonos en la Península Ibérica“. In: ATEM Archiv für Textmusikforschung 2 (2017), https://atem-journal.com/ojs2/index.php/ ATeM/article/view/2017.04/2000 (Zugriff 20.05.2018).

Herzog, Markwart (Hg.): Fußball als Kulturphänomen. Stuttgart: Kohlhammer, 2002.

Hoffmann, Thomas: „Cognitive Sociolinguistic Aspects of Football Chants: The Role of Social and Physical Context in Usage-based Construction Grammar“. In: Zeitschrift für Anglistik und Amerikanistik 63,3 (2015), 273-294.

Khodadadi, Farnosh / Gründel, Anika: Sprache und Fußball-Fangesänge. Essen: Redaktion LINSE, 2006, http://www.linse.uni-due.de/tl_files/PDF_in_Katalog/publikationen/ ESEL/Fussball_ und_Sprache_2006.pdf (10.04.2018).

Kopiez, Reinhard: „Alles nur Gegröle? Kultische Elemente in Fußball-Fangesängen“. In: Herzog, Markwart (Hg.): Fußball als Kulturphänomen. Stuttgart: Kohlhammer, 2002, 293-303.

Kopiez, Reinhard / Brink, Guido: Fußball-Fangesänge. Eine FANomenologie. Würzburg: Königshausen \& Neumann, 1998.

Küster, Rainer (2009): „Metaphern in der Sportsprache“. In: Burkhardt, Armin / Schlobinski, Peter (Hg.): Flickflack, Foul und Tsukahara. Der Sport und seine Sprache. Mannheim u. a.: Dudenverlag, 2009, 60-79.

Lavric, Eva / Pisek, Gerhard / Skinner, Andrew / Stadler, Wolfgang (Hg.): The Linguistics of Football. Tübingen: Narr Francke Attempo, 2008.

Luhrs, Joanne: „Football Chants and 'blason populaire': The Construction of Local and Regional Stereotypes“. In: Lavric, Eva / Pisek, Gerhard / Skinner, Andrew / Stadler, Wolfgang (Hg.): The Linguistics of Football. Tübingen: Narr Francke Attempo, 2008, 233-244.

Meuren, Daniel: „Im Anfang war der Rhythmus, aber der Weg zum Fan-Abitur ist weit“. In: Anstoss. Die Zeitschrift des Kunst-und Kulturprogramms zur Fifa WM 20064 (2006), 15-21.

Minonzio, Pierre-Étienne: Petit manuel musical du football. Marseille: Le mot et le reste, 2014.

Mühlbacher, Karin: „A por ellos, oé!“ Análisis literario y cultural de los cánticos y los himnos de fútbol. Saarbrücken: Editorial Académica Española, 2011.

Rosentritt, Michael: „Interview mit Volker Schlöndorff: ,Die Fans sind der antike Chor`. Der Regisseur Volker Schlöndorff über seine neue Liebe Babelsberg, sein Leiden mit Hertha und den Fußball im Film“. In: Der Tagesspiegel 17506 (23.08.2001), 28.

Rühlemann, Thorsten: „Die Fans im Stadion. Die kleinen Rituale des Alltags im Verhalten von Fußballfans“. In: Rheinisch-westfälische Zeitschrift für Volkskunde 41 (1996), 143-163.

Sacks, Harvey / Schegloff, Emanuel A. / Jefferson, Gail: „A Simple Systematics for the Organization of Turn-Taking for Conversation“. In: Language 50,4 (1973), 696-735. 
Schiering, René: „Regional Identity in Schalke Football Chants“. In: Lavric, Eva / Pisek, Gerhard / Skinner, Andrew / Stadler, Wolfgang (Hg.): The Linguistics of Football. Tübingen: Narr Francke Attempo, 2008, 221-231.

Schmidt-Lauber, Brigitta: „,Der zwölfte Mann‘. Die Europäische Ethnologie im Feld der Fußballfans“. In: Österreichische Zeitschrift für Volkskunde 112 (2009), 417-450.

Searle, John Rogers: „Eine Taxonomie illokutionärer Akte“. In: Searle, John Rogers: Ausdruck und Bedeutung. Untersuchungen zur Sprechakttheorie. Frankfurt/M.: Suhrkamp, 1982, 17-50.

Soder, Josef: Von der Fankultur im Stadion. Ein ethnologischer Blick auf das Fußballpublikum. Diplomarbeit. Universität Innsbruck 2003.

Tajfel, Henri: „Social Identity and Intergroup Behavior“. In: Social Sciences Information 13,2 (1974), 65-93.

Vanhoutte, Kristof: „The Importance of Trivial Oppositions in Football Fandom: The Narcissism of Minor Differences in Derby Games“. In: Askin, Ridvan / Diederich, Catherine / Bieri, Aline (Hg.): The Aesthetics, Poetics, and Rhetoric of Soccer. London / New York: Routledge, 2018, 126140.

Vejmelka, Marcel: „Aus der Tiefe des literarischen Raumes. Javier Marías vs. Manuel Vázquez Montalbán“. Vortrag auf der Tagung Das Spiel zwischen den Räumen. Lokale bis transnationale Dimensionen des europäischen Fußballs. Germersheim, FTSK, 20. Mai 2016. 